\title{
CENTRO Y PERIFERIA \\ EN SOCIOLOGIA DE LA EDUCACION: \\ SU DESIGUAL E INCIERTO \\ PROCESO DE INSTITUCIONALIZACION
}

Isidoro Alonso Hinojal

\section{INTRODUCCION}

El plural que vengo usando al referirme a la Sociología de la educación ${ }^{1}$ tiene varios sentidos. Uno y principal es, evidentemente, el relativo a las diversas teorías, respaldadas por las correspondientes ideologías, que, a la ruptura de la uniformidad funcionalista, fueron reapareciendo y han llegado, en general, a una cierta coexistencia; coexistencia que, de cuando en cuando, se rompe y surgen los enfrentamientos más propios de los años de su reaparición ${ }^{2}$.

Otro sentido importante del plural alude a la Sociología de la educación que se practica en diferentes países o áreas geoculturales y lingüísticas. En este sentido también cabe hablar de cierta coexistencia y de eventuales manifestaciones de tensión y enfrentamiento, pero en cualquier caso de relaciones de desigualdad. Relaciones que a veces son tan escasas que más bien parecen incomunicación, y cuando las hay resultan con frecuencia tan des-

' Alonso Hinojal, I., Educación y sociedad. Las sociologías de la educación, Madrid, C.I.S., 1980.

${ }^{2}$ Alonso Hinojal, I., "La Sociología de la educación de los setenta vista desde los ochenta". Aparecerá en Educación y Sociedad, núm. 3. 
iguales que no parece inapropiado hablar de relaciones de dominación y dependencia, o de centro y periferia para utilizar lenguaje consagrado en otros ámbitos. El estudio de estas relaciones disciplinares tiene, creo, varios núcleos de interés, entre los que destacan dos: por un lado el de añadir una vertiente más al conocimiento de la desigualdad entre sociedades poderosas y débiles, y, por otro, el de aportar también una dimensión adicional al conocimiento del estado y evolución de una especialidad en las ciencias sociales.

Estaría fuera de lugar el argumentar aquí sobre la condición de la Sociología como producto social, con rasgos propios de la sociedad que lo produce, incluso cuando en el proceso se es fiel a las exigencias epistemológicas, ni sobre la influencia de esos saberes así producidos sobre la sociedad productora. Lo que sí puede ser oportuno es destacar inicialmente dos o tres puntos relacionados con lo anterior: que las diferencias entre países o áreas culturales existen en Sociología de la educación hasta el punto de que a veces parecen poner en riesgo u ocultar los elementos comunes indispensables para ser reconocida su existencia como tal y que las relaciones, el intercambio y la construcción científica en común es a veces muy limitada.

Empezando por lo último, no deja efectivamente de sorprender que en los tiempos de la teleinformática se mantengan áreas científicas escasamente intercomunicadas. Como en otros ámbitos, está claro que no es por falta de la tecnología apropiada. La diversidad lingüística es sin duda una razón importante, razón que tiende a infravalorarse pensando que hay un par de idiomas a los que se vierte prácticamente toda la producción científica y que uno $\mathrm{u}$ otro es conocido por todo especialista. Sin olvidar las limitaciones y riesgos de ese proceder, resulta además que lo que por un lado se ve como una facilidad, puede verse por otro como un gran obstáculo, puesto que lo que no se traduce a esos idiomas corre el riesgo de permanecer prácticamente ignorado, al no pasar por los grandes circuitos internacionales de documentación.

De las barreras lingüísticas es fácil poner ejemplos ilustrativos. Bernstein ha reconocido que no pudo enterarse de los trabajos que desarrollaba el Centro Europeo de Sociología, tan próximos a los suyos en el Instituto de Educación de la Universidad de Londres, hasta que en 1975 le fueron presentados en inglés. Algo parecido se ha repetido con los trabajos de Bourdieu y colaboradores hasta que no han sido publicados en inglés diez o quince años después de haberlo sido en francés.

Más allá de los lingüísticos existen muros menos visibles pero tanto o más efectivos, como son los etnocéntricos y los ideológicos y políticos. Recordaré que un lustro o más después de haber visto la luz las obras más significativas de la llamada nueva Sociología de la educación en el Reino Uni- 
do eran recensadas en las revistas norteamericanas especializadas ${ }^{3}$. Todos ellos constituyen la negación de lo científico, y de anticientífico habría que calificar a quien por estas razones u otras prescinda de contrastar sus teorías con las demás, o de compartir sus datos con otros analistas. Las teorías no replicables y los datos no reanalizables no son científicos; el confidencialismo es anticientífico. Me da la impresión de que por estos bajos fondos se sitúa buena parte del lastre que impide navegar a las ciencias sociales.

El otro aspecto el de las diferencias nacionales o regionales, a veces parece estar a punto de romper los lazos mínimos de la unidad científica y de fraccionar la comunidad científica hasta su desaparición como tal. Esta cuestión de la comunidad científica destacada desde siempre por la Sociología del conocimiento y de la ciencia, se presenta a la vez como mucho más problemática y crucial desde la ruptura de la artificial unidad teórica de la Sociología.

Finalmente, y para entrar en materia, añadiré que, como instrumento conceptual mínimo para las comparaciones propuestas, utilizaré el concepto de institucionalización de una disciplina científica y sus cambios en los años recientes. He hecho una selección estratégica de países, y, como es de necesidad, se concede a España una especial atención.

\section{EL CENTRO}

Apoyándose en la Sociología de la ciencia, analizaba Szreter ${ }^{4}$ el proceso de institucionalización de la Sociología de la educación en la Gran Bretaña y consideraba el cumplimiento de cuatro requisitos que parece exigir tal proceso a una nueva rama del saber: el reconocimiento de esa parcela de saber en las instituciones superiores de enseñanza e investigación, normalmente mediante la creación de puestos docentes permanentes y del mayor rango; la aceptación general de la materia como campo específico de aprendizaje; la existencia de especialistas trabajando en él y la disponibilidad de una «voz pública» con la que ofrecer sus trabajos. En la Gran Bretaña estos requisitos estaban cubiertos desde hace años (Bernstein ocupó en 1967 su cátedra en la Universidad de Londres); sin embargo, dicho autor considera que no se culmina el proceso hasta 1980 en que aparece el British Journal of Sociology of Education. Este dato plantea una interesante cuestión: ¿ ¿Se trata de culminar la institucionalización de la Sociología de la educación en la Gran

${ }^{3}$ Hurn, Ch., "Recent Trends in the Sociology of Education in Britain", Harvard Educational Review, vol. 46, núm. 1, febrero 1976; APPLE, M. W., "The New Sociology of Education: Analyzing Cultural and Economic Reproduction", Harvard Educational Review, vol. 48, núm. 4, noviembre 1978.

4 SzRETER, R., "Landmarks in the Institutionalisation of Sociology of Education in Britain", Educational Review, vol. 32, núm. 3, 1980. 
Bretaña o de la institucionalización de la «nueva» Sociología de la educación?

En ese país, por supuesto, se venían y se vienen publicando trabajos de Sociología de la educación en revistas de Sociología, así como en otras dedicadas a la educación, por ejemplo, Educational Studies, desde 1975. Como reconoce un reciente análisis de las publicaciones educativas en las tres principales revistas británicas de Sociología (British Journal of Sociology, Sociological Review y Sociology), durante el último cuarto de siglo, la presencia de los temas educativos, a pesar de su bajo estatus, fue creciendo progresivamente, tanto respecto del total publicado como respecto de una destacada especialidad como es la Sociología industrial. Encuentra incluso que los temas más permanentes continuaron apareciendo en los años setenta por delante de los nuevos temas ${ }^{5}$. La aparición en 1980 del British Journal of Sociology of Education parece ser más la «voz pública» de un grupo amplio de «nuevos» sociólogos británicos de la educación, orientados u ocupados, en buena parte, en centros de formación de profesores, y acompañados de algunos colegas americanos, australianos y canadienses. Los temas y las firmas aparecidos en los cuatro volúmenes publicados así lo confirman.

No sé cómo se le ha podido escapar a tan fiel notario de la institucionalización de la disciplina en el Reino Unido una manifestación de ese proceso tan importante y significativo o más que la aparición de una revista especializada. Me refiero a las conferencias anuales de Sociología de la educación que se vienen celebrando en el Westhill College de la Universidad de Birmingham. Observador directo de la última celebrada, puedo confirmar tanto la importancia del acontecimiento, que lleva ya su sexta celebración y la sexta publicación de las correspondientes ponencias, como el carácter absolutamente predominante de los «nuevos» y jóvenes sociólogos de la educación, británicos, norteamericanos, canadienses y australianos (acompañados por la asistencia accidental de algunos «periféricos»: polacos, malteses, holandeses, españoles, etc.). Pienso que las mayores rupturas de la Sociología de la educación actual aparecen ejemplificadas en estas conferencias y esta nueva revista, tan próximas: entre generaciones, entre posiciones ideológicas, entre países y entre los especialistas ubicados en los departamentos universitarios y en centros de formación de profesores.

En lo anterior queda claro que el utilizar el esquema de la institucionalización de la materia en un país determinado no es irrelevante, sino que, por el contrario, refleja quizá de la manera más esquemática el estado de la cuestión y su evolución. Por eso utilizaré este procedimiento siempre que me sea posible y en concreto respecto de nuestro país. Antes veamos brevemente algunos otros significativos.

"SzReter, R., "Writings and Writers on Education in British Sociology Periodicals, 1953-1979", British Journal of Sociology of Education, vol. 4, núm. 2, 1983. 
Hasta donde lo permite el insuficiente conocimiento de los respectivos idiomas, dos países me parecen ejemplos de interés para mostrar el diferente nivel de implantación y desarrollo de la Sociología de la educación: Holanda y la República Federal de Alemania.

Holanda, un país pequeño pero complejo y sensible a los cambios sociales y corrientes culturales es, creo, un ejemplo de riqueza y diversidad en el desarrollo de la especialidad, como en otros muchos aspectos relacionados con la educación. Ateniéndome a un reciente informe sobre el estado de la cuestión en este país ${ }^{6}$, de los varios que existen disponibles en inglés, la especialidad se ha desarrollado y diversificado a lo largo de los años setenta, en correspondencia con las características de este país. Más concretamente, se han ampliado mucho los temas objeto de estudio; diversificado los enfoques, prestándose creciente atención a los conflictivos, fenomenológicos y del conocimiento escolar, hablándose allí de pluralidad de enfoques y no de nueva Sociología; diversificación también de las metodologías, con atención creciente a las cualitativas, pero sin abandonar las cuantitativas.

El proceso de institucionalización ha sido intenso y ofrece múltiples manifestaciones y menos conflictivas y esterilizantes de lo que algunos británicos denunciaban en aquel país. En Holanda la enseñanza de la Sociología de la educación tiene en la actualidad una fuerte demanda, tanto en los departamentos de Sociología como en los de educación; la investigación, que en los últimos años ha recibido también un fuerte estímulo en su vertiente aplicada a los diversos problemas existentes, se lleva a cabo en institutos diversificados, unos universitarios y otros no universitarios, apoyados por la importante Fundación para la Investigación Educativa (S.V.O.). Los investigadores están encuadrados en organizaciones profesionales de cooperación y periódicamente celebran encuentros varios. En Holanda no se podría decir con tanta insistencia y razón como en otras partes que los investigadores de la educación, los sociólogos entre ellos, no sean tenidos en cuenta en estos años. Un símbolo de que existe esa conexión puede serlo el que un destacado especialista, Jos van Kemenade, haya sido dos veces ministro de Educación. Decía en 1970: «Después de un lento arranque, dificultado por la tradición, la Sociología de la educación en Holanda parece estar ahora al comienzo de importantes desarrollos que van a tener amplias repercusiones tanto para la investigación como para la política educativa» ${ }^{7}$.

Aunque, evidentemente, de las consecuencias de la persistente crisis económica, de empleo y educativa no se libra hoy Holanda ni país alguno, no hay duda de que allí las circunstancias le han sido más favorables a la Sociología de la educación que en otros próximos y más poderosos como Ale-

- Wesselingh, A., "Sociology of Education in the Netherlands: situation, development, debates", British Journal of Sociology of Education, vol. 3, núm. 3, 1982.

' Citado por Wesselingh, op. cit., p. 319; cit., p. 319. 
mania Federal. Un destacado especialista de este último país, Goldsmith ${ }^{8}$, nos resume la situación: con la expansión educativa se impulsó tanto la docencia como la investigación en Sociología de la educación, dominando la influencia americana y destacando sociólogos importantes como Schelsky y Dahrendorf. En esa etapa reformista la Sociología se había, además, incorporado a la formación de los maestros según el Plan Estructural del Consejo Alemán de Educación. En la investigación educativa la Sociología jugó un papel importante aportando su propio enfoque y sirviendo de aglutinante a otras disciplinas. Entre 1963 y 1973 se crearon unos veinte institutos de investigación de carácter no universitario.

Pero al comienzo de los setenta las cosas cambiaron mucho y comenzó el retroceso de la Sociología de la educación. Las razones fueron sin duda muchas: a mediados de la década se pusieron en duda las tesis del Plan Estructural y comenzó una campaña contra la «sociologización» del magisterio, simultánea a la restauración de la autonomía pedagógica. Dirá: «La argumentación ideológica que sirve de base a la actual hostilidad frente a todo tipo de reformas - hostilidad especialmente dirigida contra la Sociologíadefiende una separación más profunda entre el contexto social de la educación, por un lado, y la orientación de los planes de estudio a materias específicas, por otro. La Sociología y la investigación sociológica ha sido acusada de pecados tan diversos como el de no haber sido capaz de prever la crisis, de trivialización de sus temas de investigación e incluso de incitación al desorden.»

Los autores no dejan de reconocer que estos cambios llegaron acompañados e impulsados por otros más amplios, como el retroceso en la reforma educativa y la falta de un enfoque unitario en la Sociología misma. "El techo común, que parecía haber sido construido para cubrir la Sociología y la investigación educativas y haber sido concluido a fines de los años sesenta con la discusión sobre problemas relativos a la socialización, comenzó muy pronto a resquebrajarse. El creciente refinamiento de métodos frente a un trasfondo de problemas metodológicos aún no clasificados, el aumento también creciente en la complejidad de los puntos de vista teóricos y, por último, la amplitud misma de las técnicas sociológicas en la investigación educativa destruyeron la relativa uniformidad de comunicación científica» ${ }^{9}$. Destacaré de esta enumeración la referencia a la ruptura de la comunidad científica, a la que vengo dando especial significación.

A un país como Francia, donde no sólo estuvo la cuna de la especialidad, sino que en los años sesenta y primeros de los setenta se produjo el renacimiento de las posiciones críticas y marxistas más influyentes, también ha

${ }^{8}$ Golaschmitd, A., y Schöfthaler, T., "La institucionalización de la Sociología de la educación en la República Federal de Alemania, 1945-1979", Educación, volumen 21.

9 Ibidem, p. 22. 
llegado la decepción para muchos. También aquí la Sociología de la educación recibió una rápida institucionalización y sus temas fueron discutidos quizá como en ningún otro país; se ha enseñado en todas partes y desde luego a los maestros. Pero ¿cuál ha sido el resultado de toda esta actividad, especialmente en cuanto medio de transformación social? La pregunta se la hacía recientemente uno de los representantes más significados de la Sociología marxista francesa, Christian Baudelot ${ }^{10}$; su respuesta significaba decepción, aunque no abandono. El resultado, si no ha sido nulo, sí ha sido mínimo. Las cosas siguen en lo fundamental como estaban, dirá. Y su propuesta hacia el futuro es mucho más científica y realista que la de otros: la Sociología de la educación debe continuar su tarea y trabajar con los maestros, pero no para decirles lo que deben hacer, sino para mostrarles «el mapa de la situación» de la escuela y para que puedan así decidir fundadamente cómo actuar.

\section{LA PERIFERIA}

Cuando en los países con tradición y mayor desarrollo en las ciencias sociales se manifiesta o acentúa este clima de decepción y cautela respecto de la Sociología de la educación sorprende ver que en otros parece que se desarrolla y consagra. Esto es lo que aparenta reflejar un número especial de Soviet Education " dedicado en 1981 a la Sociología de la educación y en el que se la considera un nuevo campo del saber incorporado a la Academia Rusa de Ciencias Pedagógicas y a dos de sus trece institutos de investigación. Para quienes no estén convencidos de que las Ciencias sociales, y la Sociología en concreto, sólo tienen sentido y valor de tales en sociedades libres, puede tener interés repasar éste $\mathrm{u}$ otros documentos análogos.

Allí se trata no de una Sociología sino de una tecnología social puesta al servicio de la planificación y sin más marco teórico que las viejas consignas seleccionadas para el momento y proclamadas por los miembros elegidos rigurosamente de entre la jerarquía política; todo ello hecho y dicho con el mimetismo propio de quienes no excluyen ninguna parcela de referencia y competición entre bloques.

La publicación aludida refleja lo acontecido en una mesa redonda celebrada en 1979 y organizada conjuntamente por la Academia Rusa de Pedagogía y la revista Sociological Research. El tema central, los protagonistas y sus repetidas recomendaciones nos ahorran mayores consideraciones. El tema y preocupación central eran, dada la caída de la natalidad en la Unión Soviética,

10 Baudelot, Ch., "La Sociología de la educación, ¿para qué?". Ponencia a las II Jornadas Nacionales de Sociología de la Educación en Escuelas Universitarias del Magisterio, Granada, mayo 1983 (no publicado).

"Soviet Education. A Journal of Translations, vol. XXIII, núm. 6, abril 1981. 
ver cómo conseguir que en los años ochenta «la juventud responda de forma entusiasta a la necesidad de ajustar su educación a su futuro trabajo» o, según los objetivos del XXV Congreso del Partido Comunista, hacer más efectiva la educación ante las crecientes demandas del socialismo desarrollado, analizando las múltiples experiencias realizadas para evaluarlas y diseminarlas en su caso. En otra dirección, se señalan determinados fenómenos negativos, como el alcoholismo, introducidos con la tecnología, la urbanización y la masificación, merecedores de la atención y el estudio.

La autocrítica no puede faltar tampoco en este ámbito educativo. Los cambios recientes han introducido innovaciones en el currículum y no todas son consideradas acertadas. "Se ha acentuado el trabajo de la memoria, pero no se han alcanzado con igual intensidad los corazones y las mentes de los alumnos.» Y esto ha alarmado al público. Por eso es natural que uno de los objetivos para el futuro trabajo de la disciplina sea «estudiar y clarificar las relaciones entre coerción y persuasión, tema extremadamente importante».

Lo anterior por sabido sobraba. Lo he incluido antes de entrar en la Sociología de la educación en los países subdesarrollados o periféricos, para poner en evidencia uno de los requisitos o precondiciones para la existencia de una Sociología que merezca tal nombre: la libertad intelectual, inseparablemente unida a las otras libertades. Y lo he traído a colación porque estos países, a los que hoy más que nunca hay que seguir llamando subdesarrollados, no disfrutan de ellas o sólo intermitentemente, con lo que las posibilidades de desarrollo científico-social se hacen también discontinuas y muy reducidas. Es el caso del llamado «cono sur» en el que, además de estar técnica y socialmente más modernizado, contaba con regimenes democráticos, hasta donde cabía, en los años cincuenta y sesenta, cuando los demás países del continente iberoamericano aún no habían podido hacer otra cosa que ensayar alguna experiencia democrática. Es bien sabido que desde el final de los años sesenta aquellas condiciones de los países del «cono sur» han desaparecido, y las que habían permitido un desarrollo de las ciencias sociales, envidiable para más de un país europeo, también. Estos cambios tan destacados no han sido los únicos, evidentemente, y obligan a esa continua transhumancia internacional e intercontinental de sus intelectuales que, aunque tenga sus consecuencias positivas, sin duda son una importante rémora para el desarrollo de las ciencias sociales, necesitadas entre otras cosas, de un trabajo continuado.

La escasez de recursos diversos y de tradición son otras tantas limitaciones tan evidentes en estos países que no es necesario pararse en ellas. Una tercera, menos manifiesta, es la que quiero aquí analizar brevemente: las relaciones, a veces las no relaciones, entre la Sociología de la educación que se realiza en estos países con la de los países más avanzados, con «el centro». 
Por supuesto, que es bien sabido y desde hace tiempo que los tres tipos de obstáculos que he citado no son independientes.

Tomaré pie para detenerme en este último aspecto, que muestra el aislamiento y ruptura de la comunidad científica en nuestra disciplina, en un reciente trabajo británico sobre el estado de la Sociología de la educación en los developing countries ${ }^{12}$.

El «subdesarrollo» total del trabajo aparece bien claro en la simple consideración de su bibliografía: de sus 25 citas, sólo tres no son en inglés, las tres en español, y sólo 11 incluyen a la educación (una sola a la Sociología de la educación), en sus enunciados. Estas «sólidas» bases le permiten entrar seguro en el tema, pues ha «elegido revisar estudios que representan los diferentes enfoques sociológicos del estudio de la educación en los países en desarrollo» (pág. 301). Así no es extraño que el trabajo se limite exclusivamente a comentar algunos autores anglosajones (además de a Gunter Frank) sobre los conceptos de desarrollo, dependencia o imperialismo cultural, o al comentario de un trabajo de análisis comparativos de los sistemas educativos de cuatro países pobres y cuatro ricos. No hay por mi parte la menor exageración; al terminar el comentario al trabajo de Williamson dirá el autor: «La última obra que quiero comentar aquí es una muy reciente». Se refiere a la de su compatriota Nash, Scbooling in Rural Societies, en la que no hay más de seis páginas dedicadas a la escuela en el medio rural de los países subdesarrollados y otras seis a los casos de Tanzania y Nigeria. Antes de comentar a estos dos autores había dedicado tres páginas a Carnoy y varias a Schultz, Gunter Frank y a Inkeles y Smith. El artículo tiene doce. Así no es extraño, sino lo más lógico, que en las pocas líneas que restan del artículo, después de los libros comentados concluya: «El enfoque sociológico de la educación en los países en desarrollo, ha sido, y continúa siendo, increíblemente estrecho» (pág. 311).

Pero quizá lo más increíble de este «informe» sea su desafortunada e irresponsable pieza «literaria» del comienzo, que no me resisto a traducir: «Para el sociólogo de la educación cuyo trabajo normalmente se limita al estudio de los sistemas educativos del oeste industrializado, entrar en la Sociología de la educación de los países en desarrollo es entrar en un paisaje intelectual extraño y turbado. Pero quizá lo más hiriente para el recién llegado es la visión de las decadentes ruinas de una civilización en otros tiempos grande y magnificente, el estructural-funcionalismo. Pero quizá igual de sorprendente sea el número de pobladores que parecen vivir felizmente y crecer boyantes en medio de los restos (muchos de ellos inconscientes de las ruinas que les rodean; ruinas que resultan tan obvias a los ojos de los extranjeros). Pero fuera de esas impresionantes ciudades que todavía parecen

12 Ball, S. J., "The Sociology of Education in Developing Countries", British Journal of Sociology of Education, vol. 2, núm. 2, 1981. 
reflejar a veces el profundo eco de sus glorias pasadas, están las junglas y las montañas, donde el imperio de la ley apenas se logra imponer. Es aquí donde las bandas rivales de guerrillas y partisanos (neo-marxistas de variadas formas) se encuentran. Sus estandartes y consignas anuncian su común oposición a la dominación por parte de los habitantes de la ciudad, pero la mayor parte de su tiempo y energías parece que se consume en rivalidades entre las propias bandas y en la lucha política entre ellas. En los más altos valles el Gobierno provisional del principal partido de la oposición (los partidarios de la perspectiva independentista) han establecido sus cuarteles. Y desde estas alturas, el país que se contempla se ofrece bien diferente. Las comunicaciones hasta aquí son pobres, las carreteras a menudo intransitables, con frecuencia se despeñan rocas y la constante erosión de los fuertes vientos hace muy traicionero el viajar y el montar cualquier cosa que no sea un campamento provisional, la más arriesgada empresa."

La cita queda para personal interpretación. El resultado sólo puede verse atenuado, además de por considerarse el autor, así lo espero, entre los recién llegados, por su confesión final: "Puede resultar también razonable preguntarse en particular y después de la revisión hecha: ¿qué ocurre con la Sociología de la educación en los países en desarrollo? Aquellos sociólogos occidentales interesados en la educación de los países en desarrollo, ¿no estarán contribuyendo al flujo del trabajo académico en una sola dirección y así inhibiendo el desarrollo de sociologías indígenas? Claramente, en el caso de América Latina, ha habido un desarrollo autónomo, la mayor parte del cual no conocemos en Europa.»

Después de lo leído, no estarían injustificados los temores de los sociólogos de la educación de los países subdesarrollados ante la recomendación final del autor, si es que alguno es capaz de pasar del párrafo transcrito. «En conjunto y tanto en Occidente como en las propias sociedades en desarrollo, hay mucho por hacer para poner la Sociología de la educación en estos países en relación viable con los más recientes avances teóricos y metodológicos logrados en los temas generales de la Sociología del desarrollo y de la educación.»

$\mathrm{Ni}$ estoy en condiciones ni es éste el lugar para elaborar un estudio de la disciplina en Iberoamérica, estados de la cuestión, por otra parte, realizados por los propios sociólogos del subcontinente, muchas veces por países. Tan sólo me permitiré un mínimo recordatorio por si en alguna medida pudiera contribuir a establecer una imagen más seria y desde una postura de intermediación entre Europa y la América iberoamericana.

- Si se piensa en manuales de Sociología de la educación, hoy son tan abundantes en Iberoamérica como en Europa; los ha habido en décadas pasadas, destacando por su fecha y por su valor reconocido internacional- 
mente el de Fernando de Azevedo, aparecido en 1940 en portugués y en 1942 en español ${ }^{13}$.

- Obras colectivas hay actuales, importantes y variadas como Mundanças Educacionais na America Latina ${ }^{14}$, coordinada por German Rama, o ya lejanas como Elites y desarrollo en América Latina, de $1967^{15}$.

- Seguro que a muchos anglosajones les suenan viejos nombres como Freire, Germani, Fals Borda, Solari o Nassif, y otros más jóvenes como German Rama, Albornoz o Graciarena. Hay que suponer que les suenan más próximos Illich y Reicner, por ejemplo.

- Es posible que, si no obras concretas, les suenen, al menos, casas editoras como Paidos, Fondo de Cultura Económica o Kapelusz. El Centro de Investigación y Desarrollo de la Educación de Chile viene publicando desde hace años los Resúmenes Analiticos de Educación, como publicación periódica de la Red Latinoamericana de Información y Documentación, y lleva ya recogidos más de 2.500 «abstracts».

- A través de las actividades de organismos como la UNESCO es posible también tener noticias del Proyecto Principal de Educación y la miltitud de trabajos a que ha dado lugar y el volumen de especialistas implicados en ellos. Algo parecido debe decirse del proyecto "Desarrollo y Educación en América Latina y el Caribe».

- Pero para orientar a quienes tengan dificultades con los idiomas tercermundistas como el español y el portugués se pueden señalar algunas pistas elementales: la revista Current Sociology dedicó ya su volumen XIX, número 1 (1971) a América Latina y en las 130 páginas de bibliografía existe un apartado dedicado a la educación.

La revista Perspectivas, que se edita también en francés e inglés, contiene frecuentes artículos relativos a Iberoamérica, y algunos números monográficos (como el número 3 del volumen VIII de 1978).

- En el último Congreso Mundial de Sociología a nadie pudo pasar desapercibida la existencia de una sesión dedicada al desarrollo educativo en América Latina con una docena de ponentes, ni la elección de un latinoamericano, Orlando Albornoz, como secretario del Comité de Investigación en Sociología de la Educación. (Habría que recordar también a Ball, que en esas ponencias aparecen más conceptos como reproducción y contradicción que los de desarrollo o dependencia.)

Entre la escueta opción teórico-ideológica de desarrollo frente a dependencia, trabajos complejos como los de German W. Rama sobre estilos edu-

13 Azevedo, F, de, Sociología de la educación, México, Fondo de Cultura Económica, 5." edición, 1961 (el reconocimiento aludido es de Roger Bastide y se relata en el Prefacio).

14 Rama, G. W. (ed.), Mudanças educacionais na America Latina, Fortaleza (Brasil), Ed. Universidade Federal do Ceará, 1983.

is Lipset, S. M., y Solari, A. E. (eds.), Elites y desarrollo en América Latina, Buenos Aires, Paidos, 1967. 
cativos, en los que se integran varios modelos en un esquema conceptual complejo, o el histórico de Gregorio Weinberg y otros muchos muestran el nivel de la Sociología de la educación en algunos de esos developing countries. Estas palabras del primero de los citados, subrayadas por el segundo, son significativas: "Participación y exclusión son los dos polos de referencia del concepto de orden que se reiteran en forma más o menos constante a lo largo de toda la historia de América Latina... y la educación, que cumple un papel central en este debate... es analizada, propugnada y combatida en términos políticos, porque lo que está en juego es saber cuáles son los valores que se deben incluir en el principio de socialización, quiénes son los sujetos que se deben socializar y quiénes constituyen las agencias sociales responsables del proceso" ${ }^{16}$. No hay que aguzar mucho el ingenio para recordar con estas palabras a los grandes animadores de la Sociología de la educación más actual: Bourdieu, Bernstein y Young.

Al recordar de nuevo el artículo de Ball habría que advertir que, conceptos como los de desarrollo y subdesarrollo u otros vistos aquí, son más que nada etiquetas peligrosas si no se colocan con el máximo cuidado. América Latina es, evidentemente, un conjunto de países, aunque con grandes diferencias entre sí, que padecen atraso económico y tecnológico. Pero esto no es todo, aun siendo tanto y tan condicionante de lo demás. Como se ve por todas partes, las manifestaciones culturales y artísticas de estos pueblos, como las de los demás, no están, por fortuna, rígidamente atadas a lo económico y lo tecnológico. La Sociología, después de todo, puede tener tanto o más que ver con el arte y desde luego con la cultura que con la economía y la tecnología ${ }^{17}$.

\section{ESPAÑA: ¿CENTRO O PERIFERIA?}

Del estado de la Sociología de la educación en España existen varios análisis, uno de ellos hecho por mí no hace mucho ${ }^{18}$. Aquí no voy a añadir uno más ni a repetir aquél; voy simplemente a actualizarlo utilizando el esquema de la institucionalización, a la vista de los cambios detectados en otros países de Europa. Antes quiero justificar estos, relativamente frecuentes, exámenes de la cuestión.

Los sociólogos han sido siempre merecedores de no pocas ironías. Se ha dicho, por ejemplo, que se pasan la vida contemplándose el ombligo, o que,

16 Rama, G. W., Educación y sociedad en América Latina y el Caribe, Santiago de Chile, UNICEF, 1980 , p. 35.

${ }^{17}$ En mi trabajo personal de hace ya más de quince años incluía más de cuatro decenas de citas latinoamericanas sobre la cuestión. Me refiero a: Alonso Hinojal, I., Los estudiantes latinoamericanos en Madrid. Orientación en los estudios $y$ actitudes hacia el cambio social, Madrid, CEDECA, 1971.

18 Alonso HinoJal, I., Educación y Sociedad, op. cit., cap. 5.3. 
mientras los demás científicos investigan, ellos discuten. Pues bien, pienso que es, no ya aceptable, sino recomendable que se discuta y revise lo que se hace, dado el fraccionamiento e incluso aislamientro entre los profesionales o grupos de ellos. La necesidad es mayor en países como el nuestro, donde no existe la comunidad de sociólogos dedicados al tema educativo, como mostraré aquí. Pienso, además, que la necesidad de autoanálisis, crítica y discusión entre representantes de diversas tendencias teóricas y líneas de trabajo metodológico, es tal que sin ellas no es posible el avance del conocimiento social. Bienvenidos, pues, esos análisis del estado de la cuestión, siempre que haya cuestión, es decir, que se haga algo más que contemplarse el ombligo.

A la vista de las últimas revisiones del estado de la cuestión en nuestro país y que luego consideraré, creo que se puede coincidir en que los años sesenta fueron los años de la iniciación de la Sociología de la educación en España y los setenta de su consolidación relativa o establecimiento propiamente tal. ¿Qué significarán los ochenta? Con los años recorridos me atrevo a proponer y discutir dos hipótesis: los ochenta no están significando su institucionalización, como se ha dicho, y están mostrando cierta decepción.

Los años sesenta, marcados por el crecimiento y la planificación económicos, vieron aparecer diversos trabajos sobre educación, realizados por economistas y administradores o, en su área de influencia, por pedagogos, psicólogos o sociólogos. Pero éstos se ocuparon de temas educativos diversos y con carácter ocasional.

Los setenta se iniciaron en educación con un acontecimiento que, la evolución y desarrollo posterior, no han permitido valorar adecuadamente. También para la Sociología de la educación es un año marcado: en el informe FOESSA de ese año se incluyó un capítulo que por su extensión y contenido destaca de los realizados hasta entonces por sociólogos; ese mismo año se celebra una mesa redonda sobre educación entre sociólogos, y luego elaborarían una obra colectiva que, sin dejar de ser variada, desigual y para algunos de los autores una aportación ocasional, es al menos significativa de la atención concedida a la especialidad; finalmente, en ese año, más o menos, empiezan a patrocinarse en los Institutos de Ciencias de la Educación y en el Centro Nacional para la Investigación y el Desarrollo de la Educación programas de becas de formación de especialistas y proyectos de investigación, que incluían la perspectiva sociológica.

Los últimos años de la década pasada y los primeros de la actual han sido testigos de acontecimientos que no es necesario recordar: en lo político, la transición a la democracia y el acceso al poder del partido socialista; en lo económico, la progresiva manifestación de la crisis, al principio «secuestrada» y luego «repercutida» por las economías más fuertes. En lo educativo, además de los fuertes efectos de los otros acontecimientos, los intentos de 
reformas, más o menos queridos, más o menos resistidos y que finalmente están en marcha: fue fácil renovar los programas de la EGB, está siendo inabordable la reforma crucial de las enseñanzas medias, se resistió hasta el agotamiento la universitaria a la que ahora se llega y que pondrá a prueba la capacidad de la propia Universidad para regenerarse a sí misma con la «autonomía multidependiente» obtenida en el año actual. $Y$ con el nuevo marco legal de la Universidad española se inicia una etapa que deberá significar mucho para la institucionalización de la Sociología de la educación en nuestro país. Sin embargo, merece la pena comprobar ya la hipótesis de la institucionalización de la disciplina en los años transcurridos y antes de que empiecen a notarse los cambios legislativos.

\section{a) INSTITUCIONALIZACIÓN Y DECEPCIÓN}

Aplícase el concepto de institucionalización a una especialidad académica ya presentado y que incluye: existencia de cátedras universitarias; estudiantes o aspirantes; "voz pública», normalmente revistas especializadas, y reuniones habituales sobre temas de la especialidad.

\section{Cátedras}

En la Universidad la primera cátedra apareció al cambio de década y de una manera poco institucional, más bien casual y personalista. Los años siguientes se dotaron varias, pero con una doble y confusa denominación, todavía no resuelta; más bien complicada con «fugas» de una a otra.

En las Escuelas Universitarias del Profesorado la situación es descrita de la siguiente manera en un documento reciente ${ }^{19}$ :

«1. Está lejos de darse (la Sociología de la educación) en todas las Escuelas y mucho más lejos de darse en forma normalizada. En algunas no existe. En la mayoría ha empezado...

2. Las condiciones de su introducción son también y en muchos aspectos irregulares.

3. Existe gran dispersión y falta de normalización en los diversos sentidos.

4. Procedencia y preparación también muy variada del actual profesorado.»

Se reclama su institucionalización, referida en general a «Normalización», lo que se justifica acudiendo a la Declaración Universal de los Derechos y a la Constitución, por un lado y al «estado actual de la ciencia sociológica en el mundo», por otro.

:4 II Jornadas Nacionales de Sociología de la Educación de Escuelas Universitarias de Magisterio, Granada, mayo de 1983, Documentación. 
Con ella implantada, o mejor «normalizada», se pretende: introducir una metodología de análisis científico del contexto social, del funcionamiento del sistema educativo, así como del papel social del maestro; desarrollar la conciencia crítica frente al «idealismo pedagogista y a cierto tecnocratismo reduccionista»; proporcionar al maestro instrumentos de análisis de la relación escuela-medio y «facilitar una transformación de la práctica profesional según el conocimiento crítico de la sociedad». En otro lugar se añade el objetivo de «ayudar a contrarrestar el aislamiento social del maestro en su trabajo», cuyo sentido queda en la ambigüedad.

Se especifican incluso los contenidos de esa pretendida normalización. La especificación muestra una imprecisa delimitación de la Sociología de la educación, pues incluye destacadamente la historia y la economía.

En todo caso, en el proceso de institucionalización de la especialidad en las Escuelas Universitarias se dan elementos de racionalidad e incipiente planificación que no existen, ni existieron que yo sepa, a nivel de Facultades.

\section{Revistas}

Planes para el lanzamiento de revistas más o menos especializadas existen desde hace tiempo, o al menos noticias frecuentes al respecto. Uno de esos intentos se ha convertido ya en realidad, la realidad del primer número, y otro se anuncia como de próxima iniciación. En la primera, que lleva por nombre Educación y Sociedad, es de destacar la participación extranjera que llega a la mitad de los artículos, reeditados por otra parte. La segunda se llamará Revista de Pedagogía Social ${ }^{20}$. Puesto que la apoyatura profesional de cada una está en Facultades Universitarias, parecen reproducir las polarizaciones y acentuar el dualismo a que antes hacía alusión.

\section{Reuniones profesionales}

Con la década actual se iniciaron varias series de reuniones que muestran aún más a las claras la ruptura y falta de delimitaciones entre ellas a que me vengo refiriendo. Creo que son diferenciables las siguientes:

\section{a) Reuniones, congresos, jornadas, etc., de las «disciplinas madre»}

a-1) De Sociología. Prácticamente en cada comunidad autónoma se va constituyendo la correspondiente asociación de Sociología que, en cuanto lo están, dan señales de vida iniciando sus respectivas reuniones periódicas. A nivel nacional se consiguió celebrar el I Congreso de Sociología en 1981. Pues bien, en todas estas reuniones se configura un grupo de Sociología de

${ }^{20}$ Educación y Sociedad, núm. 1, Madrid, Akal, primavera de 1983; Revista de Pedagogía Social, se publicará por la Sección de Ciencias de la Educación de la Universidad Autónoma de Barcelona. 
la educación cuya característica más notable es la diversidad temática, de ponentes y asistentes. En las «Conclusiones y balance» de este último acontecimiento se intenta una delimitación de tendencias que acaba siendo una buena muestra de la confusión que nos envuelve. Dice así el final del párrafo que más prometía: «De otra parte, una corriente de orígenes diversos con mayores preocupaciones teóricas y practicantes de una Sociología de la educación histórica y empírica -más antiempirista-» ${ }^{21}$.

a-2) De pedagogía. Algo parecido, pero desde la otra orilla, cabe decir de los diversos encuentros entre pedagogos. Como botón de muestra el próximo (Santiago de Compostela, julio de 1984) tiene como tema general «Educación y Sociedad plural» y como enunciado de las secciones los siguientes: Educación y pluralismo cultural y lingüístico, Educación y pluralismo ideológico y axiológico, Eduración y pluralismo socioeconómico y Educación y pluralismo político y administrativo.

\section{b) Reuniones de «la especialidad»}

b-1) Sociología de la educación (versión sociológica). Las recientes II Jornadas de Granada, ya aludidas, se consideran continuadoras de las primeras celebradas en Murcia un año antes, y ambas sucesoras del I Symposium Internacional celebrado en la Universidad Autónoma de Madrid en marzo de 1981. Estos encuentros han ido centrándose en las Escuelas de Formación del Profesorado, adoptando posturas radicales, que ya se reflejaron, y dando a los participantes extranjeros un lugar predominante.

b-2) Sociología de la educación (versión pedagogía social). Ya se han celebrado dos, uno en Sevilla en 1981 y otro en Santiago en 1982, la tercera, prevista en Barcelona, anda buscando nuevos padrino y hogar.

Como puede verse, el galimatías es difícilmente superable. Téngase en cuenta que no son éstas todas las reuniones y que en mi afán de clarificación y clasificación he simplificado al máximo y recortado flecos que, de estar presentes, las harían imposibles (es el caso de la reunión de Santiago, como hizo público el profesor Quintana Cabanas en «Cartas al Director» de El País y como dejaba ya bien claro su propio título: «II Jornadas Internacionales de Sociología de la Educación y Pedagogía Social»).

Sin necesidad de entrar en los detalles, es fácil ver un hervidero de actividades en torno a los hechos y acontecimientos que suelen considerarse como definidores de la institucionalización de una disciplina. Pero son actividades que demuestran una ausencia total de la delimitación de lo que se entiende por Sociología de la educación (salvo que se entienda que vale cualquier cosa), y muestran, en cambio, una superabundancia de tensiones e

21 I Congreso de Sociologia. Nuestra Sociología hoy. Conclusiones y balance, Zaragoza, Asociación Aragonesa de Sociología, 1982, p. 9. 
incomunicaciones que hacen totalmente fantástico pensar en la existencia de esa comunidad científica que existe, aunque dividida a veces, en otros países. La llamada, legitimadora supongo, a la participación en esos encuentros de especialistas extranjeros, de una u otra orientación, muestran los intentos de confirmar el propio enfoque, es decir, la ruptura, y la inexistencia de esa comunidad.

En resumen, creo que hay que rechazar la interpretación de que los primeros años ochenta han significado la institucionalización de la Sociología de la educación en España. A pesar de esa superabundancia relativa de signos de institucionalización, pienso que aún está por lograr la institucionalización del conflicto entre tendencias. La otra aún está más lejos.

Otro rasgo importante se detectó en los últimos años: cierta decepción, ante el rumbo de la Sociología de la educación y en general de la educación misma, por parte de quienes la miran desde las posiciones más críticas y de izquierda. Curiosamente el pesimismo de algunos autores aumenta con el logro de los importantes objetivos políticos de esos mismos grupos políticos: implantación de la democracia y acceso al poder de los socialistas. Es una especie de nostalgia de las luchas más o menos clandestinas y de decepción prematura dada la inmediatez de estos cambios políticos ${ }^{22}$.

Un reflejo claro y variado de esa actitud pesimista respecto de la educación y la propia Sociología de la educación por parte de la izquierda radical, crítica del poder y la política socialista, lo ofrece el número centenario de Cuadernos de Pedagogía. Gómez Llorente cree que todo lo que criticaba cuatro años atrás y todo lo que entonces había de eliminar de la educación aún sigue ahí; hacia el futuro "está por ver qué va a ocurrir». Jordi Monés es aún más explícito: «estamos asistiendo a una clara desvirtuación de los planteamientos democráticos del período $1970-77 \ldots$ Todo parece indicar que se marcha hacia una racionalización global del sistema educativo». Respecto de las enseñanzas sobre educación se detecta, dice, un marcado utilitarismo en los estudios de magisterio y un excesivo teoricismo en los de ciencias de la educación. Sin duda la clave de este pesimismo teórico está en «el declive del pensamiento educativo marxista, principalmente por lo que hace referencia a las relaciones escuela-trabajo productivo" (pág. 65).

Para la Sociología de la educación esta misma actitud la refleja Marina Subirat. A comienzos de los setenta se logra una «debilísima implantación», pero con «inicios de vitalidad» que se van confirmando puesto que la Sociología de la educación se afianza hacia mediados de la década; pero a finales de ella «el tono vital empieza a decaer», precisamente «a partir de unas primeras elecciones democráticas que pudieron haber sido el umbral de una

${ }^{22}$ Destacan estas posturas en: Drtega, F., "Pasado y presente de la Sociología de la educación en España", en Perspectivas actuales en Sociologia de la educación, Madrid, Ed. Cantoblanco (Universidad Autónoma de Madrid), 1983; SubIRAt, M., "La Sociología de la educación", Cuadernos de Pedagogía, núm. 100. 
reconstrucción del sistema escolar, y no fueron, en este ámbito, sino la continuación de una rutina, agravada por la pérdida de los proyectos y movilizaciones anteriores». Así pues, la atonía de la Sociología de la educación tendrá también para Subirat esas causas externas, en la situación de la propia educación, y otras propias e internas de la Sociología: su raquitismo institucional en Facultades y Escuelas Universitarias y el carácter esporádico de la investigación (la «manía investigadora» de algunas personas). Pero hay un segundo elemento, éste teórico y sin duda más grave en la presente coyuntura educativa: «una vez establecido el hecho -incontestable - de que la escuela reproduce las jerarquías sociales ¿para qué seguir investigando?» (pág. 83).

\section{c) Orientación de los trabajos}

Aun sin intentar un análisis detallado de la producción publicada desde mi anterior consideración de la situación, un somple repaso a las más recientes publicaciones individuales ${ }^{23}$ y colectivas $^{24}$ pueden permitir delinear la orientación más reciente de la Sociología de la educación en nuestro país.

A pesar de que los trabajos a la vista no son muy numerosos, su enorme diversidad hace difícil su clasificación e incluso la elección de los criterios con los que confeccionarla. Elijo, como salida, el continuo teórico-empírico.

1. Trabajos teóricos. Son, sin duda, los predominantes. Su contenido y forma son tan variados que cabría incluso descartar como no científicos algunos: los de carácter periodístico y las «elucubraciones alucinógenas», por ejemplo. De los restantes, alguno podría considerarse como ensayo precientífico o exploratorio, cuando, pongo por caso, se elabora un tema sin referencia alguna a otras aportaciones o estudios anteriores. Aún cabría diferenciar los trabajos teóricos de los metateóricos y los actuales de los históricos.

2. $\quad$ Trabajos teórico-empíricos. Su variedad, además de temática, está en la proporción y grado de integración entre parte teórica y empírica; en unos predominará la teoría, en otros los datos, pero en todos ellos están presentes los dos componentes y se ha establecido entre ellos la relación mínima que exige la metodología. Por supuesto, una y otra parte cumplen unas exigencias mínimas. Constituirían la investigación empírica propiamente tal.

3. Algunos trabajos habría que calificarlos de descriptivismo ateórico o «empirismo abstracto»; es decir, la simple recogida y acumulación de datos sin un marco teórico o justificación suficiente. Algunos pueden incluso im-

${ }^{23}$ Entre las individualidades más recientes están: LERENA, C., Reprimir y liberar. Critica sociológica de la educación y la cultura contemporáneas, Madrid, Akal, 1983; Moncada, A., Más allá de la educación, Madrid, Tecnos, 1983; Colom CañeLlas, A. J., Teoría y metateoria de la educación. Un enfoque a la luz de la teoria general de sistemas, México, Trillas, 1982; Fernández de Castro, I., Sistema de enseñanza y democracia, Madrid, Siglo XXI, 1980.

${ }^{24}$ Las dos publicaciones colectivas son: Perspectivas actuales en Sociología de la educación, Madrid, Ed. Cantoblanco (Universidad Autónoma), 1983; Educación y Sociedad, revista publicada por Akal, núm. 1, 1983. 
plicar el uso de la moderna tecnología del cálculo, lo que evidentemente no le añade ningún mérito.

4..$^{\circ}$ Quedan, finalmente, algunos trabajos cuyo destino lógico hubiera sido el grupo $2^{\circ}$, pues incluyen teoría y datos. Pero la teoría es más o menos ensayista y los datos más o menos ingenuos, pero una y otros desconectados totalmente, aunque se pretenda justificar lo uno con lo otro. Más que trabajos del tipo $2 .^{\circ}$ son trabajos parciales yuxtapuestos del tipo $1 .^{\circ}$ y $3 .^{\circ}, \mathrm{y}$ como tales de valor engañoso y casi nulo.

El avance del conocimiento de lo educativo depende fundamentalmente de trabajos del tipo $2 .^{\circ}$ y de algunos del tipo $1 .^{\circ}$; muy poco de los restantes.

Resumiendo este repaso, la Sociología de la educación en España en los años recientes, los primeros de la nueva década, no ha logrado institucionalizarse y parece lejos de ello, ya que ni siquiera ha institucionalizado los fuertes conflictos entre grupos y tendencias. Sin embargo y para lograrlo ha realizado, está realizando o piensa realizar, una serie de actuaciones (reuniones, publicaciones, cátedras, etc.) excesivas, comparando nuestro nivel científico y el de otros países, y que pueden dificultar aún más el proceso, dado que intentan consagrar a grupos o tendencias aisladas, excluyendo a las demás. Las tensiones y falta de comunicación entre unos y otros impide la existencia mínima de una comunidad de sociólogos de la educación, a lo que contribuye, y ha contribuido siempre, el estado de la Sociología en nuestro país. También resulta desproporcionada la clara decepción de algunos sectores que, comparada con la que hemos constatado en otros países, parece aquí prematura y más negativa. Los trabajos sin duda han seguido aumentando en número y variedad en los últimos años; siguen siendo abundantes los de difícil consideración científica y predominantes los teóricos de un signo u otro. En los más convencionales, es lenta la incorporación de las exigencias metodológicas de uso hoy generalizado.

Hacia el futuro, y más allá de esos pesimismos aludidos, dos acontecimientos actuales van a ser decisivos, además de la influencia más general de circunstancias políticas y limitaciones económicas, sin duda seguras: el desarrollo y aplicación de la reforma universitaria y el ejercicio autonómico de las facultades en proceso de transferencia en materia educativa (enseñanza e investigación). El desarrollo real de la reforma universitaria aprobada afectará directa y decisivamente a las pretensiones de institucionalización de la especialidad en sus más destacados niveles (Facultades, Escuelas e Institutos universitarios), logro que deberá antes pasar por la institucionalización de los conflictos, hoy casi salvajes.

Si nos comparamos con los países próximos, el resultado no parece muy satisfactorio: al tradicional retraso y dependencia colonial, hay que añadir esos ramalazos de decepción anticipada, y ese desgaste en actividades superabundantes de seudoinstitucionalización y enfrentamiento, más escandalosos cuando los recursos se hacen más escasos. 
NOTAS 